\title{
Adaptação transcultural para o idioma português do Cocaine Craving Questionnaire - Brief
}

\author{
Transcultural adaptation into Portuguese language of the Cocaine Craving Questionnaire - Brief
}

\author{
Renata Brasil Araujoํ, Rosemeri Siqueira Pedroso², Maria da Graça Tanori de Castro³ \\ 1 Doutora em Psicologia pela Pontifícia Universidade Católica do Rio Grande do Sul (PUC-RS). \\ 2 Psicóloga, mestre em Psicologia Clínica pela PUC-RS. \\ 3 Psiquiatra, mestre em Psicologia Clínica pela PUC-RS
}

Recebido: 3/8/2009 - Aceito: 30/9/2009

\begin{abstract}
Resumo
Contexto: A avaliação do craving (ou fissura) é muito importante no tratamento de dependentes de cocaína, sendo necessárias adaptações transculturais de escalas para aumentar a efetividade na avaliação e no tratamento dessa clientela. Objetivo: O objetivo deste estudo foi traduzir, adaptar culturalmente e verificar a equivalência semântica do Cocaine Craving Questionnaire - Brief(CCQ-Brief), o qual avalia o craving (fissura) por cocaína. Método: O CCQ-Brief foi traduzido do inglês para o português, aplicado em 10 sujeitos e submetido ao brainstorming em um grupo de 4 sujeitos para reprodução individual e verbal, item a item. Realizou-se o backtranslation da primeira tradução e do brainstorming. Logo após, traduziu-se novamente para o português. Todo o processo foi analisado por um comitê de juízes especialistas, os quais emitiram pareceres com as observações pertinentes. Resultados: Considerando-se os pareceres dos especialistas e um último estudo piloto, construiu-se a versão final brasileira do CCQ-Brief e uma versão adaptada dessa escala para usuários de crack. Conclusão: Os resultados indicaram uma equivalência semântica satisfatória entre as versões, podendo o CCQ-Brief ser útil no tratamento de dependentes de cocaína.
\end{abstract}

Araujo RB, et al. / Rev Psiq Clín. 2010;37(5):195-8

Palavras-chave: Estudos de validação, cocaína, cocaína crack.

\begin{abstract}
Background: The evaluation of craving is very important to treatment of cocaine dependence and the cross-cultural adaptations of scales are needed for increase effectiveness in the evaluation and treatment of them. Objective: The objective of this study was to translate, to adapt culturally and verify the semantic equivalence of the Cocaine Craving Questionnaire - Brief (CCQ-Brief), which evaluates the craving for cocaine. Method: The CCQ-Brief was translated from English to Portuguese, administered to 10 persons, and then submitted to brainstorming in a group of 4 persons for individual and verbal reproduction, item by item. Backtranslation was executed based on first translation and from brainstorming to the origin language. Soon after, it was translated again into Portuguese. All the process was analyzed by a committee of specialists, which emitted a decision and the pertinent comments. Results: Considering the decision of the specialists and one last Pilot study, was constructed of the final Brazilian version of the CCQ-Brief and the version adapted that scale for crack users. Discussion: Results indicated a satisfactory semantic equivalence between versions and the CCQ-Brief that may be important in the treatment of the cocaine dependents.
\end{abstract}

Araujo RB, et al. / Rev Psiq Clín. 2010;37(5):195-8

Keywords: Validation studies, cocaine, crack cocaine.

\section{Introdução}

O craving ou "fissura" é um conceito controverso na literatura científica ${ }^{1}$. Alguns autores defendem a definição mais comum de que ele é um intenso desejo de utilizar uma determinada substância ${ }^{2-5}$, outros, no entanto, consideram importante entendê-lo de forma multidimensional, que inclui nesse conceito não só o desejo pelo uso da droga, mas também a capacidade de essa substância aliviar os sintomas de abstinência ou o afeto negativo, a expectativa de resultado positivo, a intenção de usar ${ }^{1,6-9}$ e a perda de controle a respeito desse desejo ${ }^{10}$.

Pelo fato de o craving ser uma variável fundamental no tratamento de dependentes químicos, houve uma preocupação da comunidade científica com a elaboração e a validação de instrumentos para mensurá-lo6-9,11,12. O CCQ-Brief, desenvolvido por Sussner et al. ${ }^{13}$, é uma escala de 10 questões elaborada a partir das 45 questões do Cocaine Craving Questionnaire Now, elaborado por Tiffany et al. ${ }^{10}$, o qual foi validado no Brasil por Silveira et al. ${ }^{14}$. O CCQ-Brief é uma escala likert de 7 pontos, que vai de "discordo totalmente" até "concordo totalmente". Na versão de 45 itens (CCQ), a escala foi dividida em cinco categorias: Desejo de usar cocaína, Antecipação de resultado positivo, Alívio dos sintomas da abstinência ou afeto negativo, Intenção e Planejamento para o uso da cocaína e Falta de controle desse uso; no entanto, a escala abreviada não obteve essa subdivisão. O escore do CCQ-Brief é obtido a partir da soma de pontos de cada questão, sendo um escore total.

A validação psicométrica do CCQ-Brief foi realizada com uma amostra de 247 indivíduos internados para tratar a dependência química, que preenchiam critérios para abuso ou dependência de cocaína de acordo com o DSM-IV. Na verificação da consistência interna da escala, obteve-se um valor de $\infty=0,90$, tendo sido encontrada uma correlação positiva do CCQ-Brief com a versão longa da escala, o CCQ-Now ${ }^{13}$, demonstrando que esse é um instrumento adequado para mensurar o craving.

O craving é, sem dúvida alguma, uma das variáveis que dificulta a manutenção da abstinência em dependentes químicos e deve ser estudado para que sejam diminuídas as altas taxas de recaída dessa clientela ${ }^{6,15,16}$. Assim, o objetivo deste estudo é apresentar a adaptação transcultural para o português do CCQ-Brief ${ }^{13}$ para a avaliação do craving pela cocaína e pelo crack. 


\section{Método}

Após a autorização dos autores da versão em inglês da escala CCQBrief ${ }^{13}$, foi iniciado o processo de sua adaptação transcultural para o português, a qual foi norteada por estudos anteriores como os de Araujo et al. $.6,7$, Mattos et al. ${ }^{17}$ e Pedroso et al. ${ }^{18,19}$.

As etapas do processo de validação foram:

Etapa 1: Tradução do CCQ-Brief da língua inglesa para a língua portuguesa, realizada por uma professora de inglês, com bacharelado em Letras, habilitada na língua inglesa e conhecedora do objetivo da tradução.

Etapa 2: Aplicação do instrumento traduzido a 10 sujeitos, dependentes de cocaína inalada, de acordo com a CID-1020, abstinentes desta substância há pelo menos um mês, em tratamento em um ambulatório especializado em dependência química, com o objetivo de analisar se as questões eram de fácil compreensão, levantando possíveis dúvidas.

Etapa 3: Brainstorming - foram reunidos 5 sujeitos, profissionais que trabalhavam com dependência química, os quais deveriam reproduzir verbalmente cada questão que compõe o instrumento, sendo investigada a compreensão delas.

Etapa 4: Backtranslation - a primeira tradução dos instrumentos foi revertida para o idioma de origem (inglês) por um nativo de língua inglesa, fluente na língua portuguesa e que não conhecia o objetivo da tradução.

Etapa 5: Tradução do instrumento - a partir do backtranslation, as duas versões foram novamente traduzidas para a língua portuguesa por uma psicóloga, brasileira, com fluência na língua inglesa e que conhecia a finalidade da tradução.

Etapa 6: Nesta fase da validação, foi composto um comitê de 3 juízes - 3 psiquiatras, todos com formação teórica e prática em dependência química. $\mathrm{O}$ trabalho desses profissionais consistia em comparar as versões dos instrumentos, verificando se os itens dos questionários referiam-se ou não ao tema "craving", bem como opinar a respeito de quais itens das duas versões traduzidas para a língua portuguesa eram mais adequados à finalidade dos instrumentos. Os resultados devolvidos pelos membros do comitê foram levantados, sendo produzidas as versões finais dos instrumentos.

Etapa 7: Estudo piloto - a versão final em português foi aplicada a uma amostra composta por 20 pacientes dependentes de cocaína internados para desintoxicação em uma unidade masculina especializada em dependência química, sendo o diagnóstico realizado segundo os critérios da CID-1020. Desses, 10 faziam uso de cocaína fumada (crack), 5, de cocaína inalada, e 5, de injetável. Era critério de exclusão apresentar sintomas psicóticos ou ser analfabeto.

O objetivo desta aplicação foi verificar, do ponto de vista desses pacientes, se o instrumento estava adequado e se era de fácil compreensão.

Etapa 8: A partir do resultado da etapa 7, foi observado que a escala, com algumas modificações relativas à forma do enunciado, estava adequada para quem utilizava a cocaína pela via inalada e injetável, mas que não era tão clara para os que a utilizavam pela via fumada ( crack). Assim, foi decidido manter essa versão da tradução (apenas com as mudanças no enunciado), mas também adaptá-la para a mensuração do craving pelo crack.

Etapa 9: Adaptação da versão em português do CCQ-Brief para aplicação em usuários de crack por uma psicóloga que trabalha com dependentes de crack e correção dessa versão por uma professora de língua portuguesa, com licenciatura em Letras.

Etapa 10: Aplicação da versão para usuários de crack da escala a 10 dependentes de crack e da versão final da escala original a 10 dependentes de cocaína inalada, diagnosticados de acordo com o CID-1020. Todos estavam no sétimo dia de abstinência dessa substância e deveriam avaliar se os instrumentos estavam adequados tanto em termos de compreensão quanto de funcionalidade.

\section{Aspectos éticos}

O início do processo de validação do CCQ-Brief ${ }^{13}$ ocorreu a partir do envio do instrumento e da autorização dos autores para esta pesquisa, via correio eletrônico.
Somente após o projeto de pesquisa ser aprovado pelo Comitê de Ética em Pesquisa do Hospital Psiquiátrico São Pedro, foi iniciada a parte da adaptação transcultural que incluía aplicação do instrumento a pacientes ou a outros seres humanos. Antes de cada indivíduo aceitar fazer parte da pesquisa, foi explicada a finalidade dela, sendo fornecido um Termo de Consentimento Livre e Esclarecido, cuja assinatura era uma pré-condição para a participação.

\section{Resultados}

Nas etapas 1, 2 e 3, pode-se observar que a escala foi compreendida, não sendo levantadas dúvidas pelos dependentes nem pelos profissionais. Os comentários foram positivos e foi salientada a rapidez de sua aplicação (média de 4 minutos).

Um exemplo das etapas 1,4 e 5 - o processo de tradução do enunciado da escala - pode ser observado na tabela 1 .

Após a etapa 6, analisando os comentários dos juízes, foi decidido:

- Manter o nome original da escala, acrescentando suas iniciais - CCQ-B -, e a expressão "Versão Brasileira" no final, para facilitar a busca em bancos de dados internacionais. Também se optou por utilizar o enunciado da tradução da versão original, já que houve consenso dos 3 juízes com relação a esse aspecto.

- A questão 1, que na versão original foi traduzida como: "Quero tanto cocaína que quase posso prová-la”, seria substituída por "Eu desejo tanto usar cocaína que quase posso sentir o seu gosto". As autoras substituíram "quero" por "desejo" para dar mais a ideia de craving, que é o objetivo da questão, acrescentaram o verbo "usar", por não ser comum o uso dessa frase sem verbo pelos usuários de cocaína, e trocaram "prová-la" por "sentir seu gosto", porque, na tradução, a primeira frase dá ideia de ação, o que não é o objetivo da questão. A escolha por "sentir o gosto" tem relação com esta ser uma expressão muito usada por dependentes de cocaína no Brasil e que se assemelha ao sentido que os autores quiseram dar à questão.

- Na questão 2, a expressão "desejo muito grande" foi substituída por "desejo muito forte".

- As questões 3, 6, 7 e 9 permaneceram iguais às da tradução do original, conforme consenso dos juízes, e as questões 4 e 10 foram modificadas apenas na troca da gíria "coca" da americana para o termo "cocaína" na versão brasileira. $\mathrm{Na}$ questão 5 também foi feita essa última troca, mas a expressão "Tenho fissura por coca agora" foi substituída por "Eu estou com fissura por cocaína agora". A troca de "coca" por "cocaína" foi sugerida por 2 juízes, e as autoras, por considerarem "coca" uma gíria que pode sofrer influências regionais, decidiram acatar essa sugestão.

- Na questão 8, a expressão "Usar cocaína agora faria com que as coisas parecessem perfeitas" na tradução da versão original foi substituída por "Usar cocaína agora faria as coisas parecerem perfeitas".

- Na etapa 7 - o estudo piloto -, os dependentes demonstraram ter alguma dificuldade (o que não havia ocorrido na etapa 2) com o enunciado da escala, pois ela apresentava, em cada questão, 7 traços separados por dois pontos entre concordo totalmente e discordo totalmente, o que causava confusão para alguns (Tabela 1). Por isso, as autoras preferiram, na etapa 8 , colocar em cima dos traços em branco números que corresponderiam ao grau de craving, facilitando, assim, sua compreensão. Essa versão final foi adaptada para seu uso em dependentes de crack, na etapa 9, em virtude das dúvidas dos sujeitos durante a aplicação, durante a qual, frequentemente, perguntavam se "cocaína queria dizer crack".

- As duas versões da escala, na etapa 10, foram entendidas sem dificuldade por todos os sujeitos, sendo o tempo médio de aplicação o de 3 minutos, um pouco inferior ao da etapa 1 , talvez por causa da mudança no enunciado. 
- Na adaptação para os usuários de crack, na versão final, foram substituídos os termos "cocaína" por "crack", ou a expressão "usar cocaína" por "fumar crack", sendo também substituída a preposição "por" (como no exemplo: desejo "por" cocaína) pela contração "pelo" (desejo "pelo" crack), segundo sugestão da professora de língua portuguesa. No nome da escala foi acrescentado "Versão Brasileira": "Adaptada para o Crack". As versões finais dos instrumentos estão dispostas nas tabelas 2 e 3.

\section{Discussão}

O craving é um dos determinantes mais importantes da recaída em dependentes de substâncias psicoativas ${ }^{21}$, entre elas, a cocaína ${ }^{1}$; assim, elaborar e validar instrumentos mais fidedignos para avaliálo é de fundamental relevância na área clínica ${ }^{6,7,9,11,12,14}$. O cuidado metodológico neste processo, objetivando a validação semântica e psicométrica dos instrumentos, é de extrema importância para a obtenção de métodos fidedignos para a avaliação de diferentes variáveis ${ }^{17-19,22}$.

O entendimento do craving como um construto multidimensional torna o uso de instrumentos unidimensionais, como as escalas analógico-visuais que mensuram "desejo" como único fator, uma forma muito simplificada para explicar esse fenômeno ${ }^{1}$. Assim, a adaptação transcultural do CCQ-Brief ${ }^{13}$, em um momento em que se observa aumento da prevalência de dependentes de crack/cocaína em amostras clínicas ${ }^{23,24}$, pode ser muito útil ao ser delineado o plano terapêutico dessa clientela, com vistas a monitorar uma variável que, se controlada, pode aumentar as taxas de manutenção da abstinência6,15,16. O CCQ-Brief - Versão Brasileira e sua versão adaptada para o crack obtiveram um excelente nível de equivalência

Tabela 1. Etapas de tradução, backtranslation, tradução 2 e da elaboração da versão final do enunciado do CCQ-B

\begin{tabular}{|c|c|c|c|c|}
\hline Versã & Tradução 1 & Backtranslation & Tradução 2 & Versão final \\
\hline $\begin{array}{l}\text { Cocaine Craving } \\
\text { Questionnaire-Brief } \\
\text { Indicate how much you agree } \\
\text { or disagree with each of } \\
\text { the following statements by } \\
\text { placing a single check mark } \\
\text { (like this:_V__lalong each line } \\
\text { between STRONGLY DISAGREE } \\
\text { and STRONGLY AGREE. The } \\
\text { closer you place your checkmark } \\
\text { to one end or the other } \\
\text { indicates the strength of your } \\
\text { disagreement or agreement. } \\
\text { Please complete every item. } \\
\text { We are interested in how you } \\
\text { are thinking or feeling right } \\
\text { now as you are filling out the } \\
\text { questionnaire }\end{array}$ & $\begin{array}{l}\text { Questionário B sobre Fissura } \\
\text { em Cocaína } \\
\text { Indique o quanto você } \\
\text { concorda ou discorda com } \\
\text { cada uma das frases abaixo } \\
\text { marcando em apenas um dos } \\
\text { espaços (exemplo:_VI) entre } \\
\text { DISCORDO TOTALMENTE e } \\
\text { CONCORDO PLENAMENTE. } \\
\text { Quanto mais próxima for a } \\
\text { marca de um dos lados, mais } \\
\text { você concordará ou discordará } \\
\text { da frase. Por favor, complete } \\
\text { cada item. Gostaríamos de } \\
\text { saber o que você pensa e sente } \\
\text { agora enquanto responde ao } \\
\text { questionário }\end{array}$ & $\begin{array}{l}\text { uestionnaire B on Cocaine } \\
\text { aving } \\
\text { how how much you agree } \\
\text { disagree with each one } \\
\text { the sentences below by } \\
\text { ecking only one of the spaces } \\
\text { xample:_V_l between } \\
\text { RONGLY DISAGREE and } \\
\text { RONGLY AGREE. The closer } \\
\text { one of the ends is your } \\
\text { ark, the stronger you agree } \\
\text { disagree with the sentence. } \\
\text { ease complete each item. We } \\
\text { ould like to know what you } \\
\text { e thinking and feeling right } \\
\text { ow while you complete the } \\
\text { eestionnaire }\end{array}$ & $\begin{array}{l}\text { io B sobre Fissura } \\
\text { a } \\
\text { lanto você concorda } \\
\text { de cada uma das } \\
\text { abaixo marcando } \\
\text { t espaço (exemplo: } \\
\text { DISCORDO } \\
\text { TE e CONCORDO } \\
\text { TE. O quanto mais } \\
\text { e aproximar das } \\
\text { es, mais você } \\
\text { discorda com a } \\
\text { Vós gostaríamos de } \\
\text { você está pensando } \\
\text { gora enquanto } \\
\text { se questionário }\end{array}$ & $\begin{array}{l}\text { raving } \\
\text { aire - Brief } \\
\text { Versão Brasileira } \\
\text { uanto você concorda } \\
\text { com cada uma das } \\
\text { xo marcando em } \\
\text { dos números entre } \\
\text { TOTALMENTE e } \\
\text { TOTALMENTE. } \\
\text { is próxima for a } \\
\text { m dos lados, mais } \\
\text { rdará ou discordará } \\
\text { or favor, complete } \\
\text { Gostaríamos de } \\
\text { você pensa e sente } \\
\text { uanto responde ao } \\
0\end{array}$ \\
\hline
\end{tabular}

Tabela 2. Cocaine Craving Questionnaire - Brief (CCQ-B) - Versão Brasileira

\begin{tabular}{l} 
Cocaine Craving Questionnaire - Brief(CCQ-B)-Versão Brasileira \\
Indique o quanto você concorda ou discorda de cada uma das frases abaixo \\
marcando em apenas um dos números entre DISCORDO TOTALMENTE e \\
CONCORDO TOTALMENTE. Quanto mais próxima for a marca de um dos lados, \\
mais você concordará ou discordará da frase. Por favor, complete cada item. \\
Gostaríamos de saber o que você pensa e sente agora enquanto responde ao \\
questionário. \\
\hline 1. Eu desejo tanto usar cocaína que quase posso sentir seu gosto. \\
DISCORDO TOTALMENTE $1: 2: 3: 4: 5: 6: 7$ CONCORDO TOTALMENTE \\
2. Eu tenho um desejo muito forte por cocaína. \\
DISCORDO TOTALMENTE $1: 2: 3: 4: 5: 6: 7$ CONCORDO TOTALMENTE \\
3. Vou usar cocaína assim que puder. \\
DISCORDO TOTALMENTE $1: 2: 3: 4: 5: 6: 7$ CONCORDO TOTALMENTE \\
4. Acho que poderia resistir a usar cocaína neste momento. \\
DISCORDO TOTALMENTE $1: 2: 3: 4: 5: 6: 7$ CONCORDO TOTALMENTE \\
5. Eu estou com fissura por cocaína agora. \\
DISCORDO TOTALMENTE $1: 2: 3: 4: 5: 6: 7$ CONCORDO TOTALMENTE \\
6. Tudo que queria fazer agora era usar cocaína. \\
DISCORDO TOTALMENTE $1: 2: 3: 4: 5: 6: 7$ CONCORDO TOTALMENTE \\
7. Não sinto nenhum desejo por cocaína neste momento. \\
DISCORDO TOTALMENTE $1: 2: 3: 4: 5: 6: 7$ CONCORDO TOTALMENTE \\
8. Usar cocaína agora faria as coisas parecerem perfeitas. \\
DISCORDO TOTALMENTE $1: 2: 3: 4: 5: 6: 7$ CONCORDO TOTALMENTE \\
9. Eu vou usar cocaína assim que tiver a chance. \\
DISCORDO TOTALMENTE $1: 2: 3: 4: 5: 6: 7$ CONCORDO TOTALMENTE \\
10. Nada seria melhor do que usar cocaína agora. \\
DISCORDO TOTALMENTE $1: 2: 3: 4: 5: 6: 7$ CONCORDO TOTALMENTE
\end{tabular}

Tabela 3. Cocaine Craving Questionnaire-Brief(CCO-B)-Versão Brasileira Adaptada para o Crack

\begin{tabular}{l} 
Cocaine Craving Questionnaire - Brief(CCO-B) - Versão Brasileira Adaptada \\
para o Crack \\
Indique o quanto você concorda ou discorda de cada uma das frases abaixo \\
marcando em apenas um dos números entre DISCORDO TOTALMENTE e \\
CONCORDO TOTALMENTE. Quanto mais próxima for a marca de um dos lados, mais \\
você concordará ou discordará da frase. Por favor, complete cada item. Gostaríamos \\
de saber o que você pensa e sente agora enquanto responde ao questionário. \\
\hline 1. Eu desejo tanto fumar crack que quase posso sentir seu gosto. \\
DISCORDO TOTALMENTE $1: 2: 3: 4: 5: 6: 7$ CONCORDO TOTALMENTE \\
2. Eu tenho um desejo muito forte pelo crack. \\
DISCORDO TOTALMENTE $1: 2: 3: 4: 5: 6: 7$ CONCORDO TOTALMENTE \\
3. Vou fumar crack assim que puder. \\
DISCORDO TOTALMENTE $1: 2: 3: 4: 5: 6: 7$ CONCORDO TOTALMENTE \\
4. Acho que poderia resistir a fumar crack neste momento. \\
DISCORDO TOTALMENTE $1: 2: 3: 4: 5: 6: 7$ CONCORDO TOTALMENTE \\
5. Eu estou com fissura pelo crack agora. \\
DISCORDO TOTALMENTE $1: 2: 3: 4: 5: 6: 7$ CONCORDO TOTALMENTE \\
6. Tudo que queria fazer agora era fumar crack. \\
DISCORDO TOTALMENTE $1: 2: 3: 4: 5: 6: 7$ CONCORDO TOTALMENTE \\
7. Não sinto nenhum desejo pelo crack neste momento. \\
DISCORDO TOTALMENTE $1: 2: 3: 4: 5: 6: 7$ CONCORDO TOTALMENTE \\
8. Fumar crack agora faria as coisas parecerem perfeitas. \\
DISCORDO TOTALMENTE $1: 2: 3: 4: 5: 6: 7$ CONCORDO TOTALMENTE \\
9. Eu vou fumar crack assim que tiver a chance. \\
DISCORDO TOTALMENTE $1: 2: 3: 4: 5: 6: 7$ CONCORDO TOTALMENTE \\
10. Nada seria melhor do que fumar crack agora. \\
DISCORDO TOTALMENTE $1: 2: 3: 4: 5: 6: 7$ CONCORDO TOTALMENTE \\
\hline
\end{tabular}


semântica com a escala original em língua inglesa. As maiores dificuldades encontradas foram quanto à compreensão do enunciado e à sua adaptação para usuários de crack, o que foi corrigido a partir da construção de duas versões distintas.

As autoras procuraram não utilizar gírias nas escalas por estas sofrerem influências regionais e poderem ser modificadas com o passar do tempo, algo tão comum nas expressões usadas por dependentes químicos.

Pode-se observar o quanto foi importante, no processo de adaptação transcultural, as aplicações da escala a sujeitos dependentes de cocaína/crack para uma avaliação mais precisa de seu nível de objetividade e clareza para mensurar o craving.

Deve-se ressaltar que a adaptação transcultural é o primeiro passo de todo o processo de validação de uma escala ${ }^{22}$ e que já foi iniciada pelas autoras, em uma amostra de pacientes internados para desintoxicação, a validação psicométrica das versões brasileiras do CCQ-Brief, sendo importante que os leitores tenham cautela, antes da publicação destes resultados, na aplicação delas.

\section{Agradecimentos}

Ao Dr. Bradley Sussner e ao Dr. Stephen Tiffany, pela permissão de apresentar o CCQ-Brief - Versão Brasileira e o CCQ-Brief - Versão Brasileira Adaptada para o Crack neste artigo. Ao referir-se às versões brasileiras do CCQ-Brief, solicitamos que sejam citados os artigos Tiffany et al. ${ }^{10}$ e Sussner et al. ${ }^{13}$ encontrados nas referências deste artigo.

\section{Referências}

1. Araujo RB, Oliveira MS, Pedroso RS, Miguel AC, Castro MGT. Craving e dependência química: conceito, avaliação e tratamento. J Bras Psiquiatr. 2008;57(1):57-63.

2. Beck AT, Wright FD, Newman CF, Liese BS. Cognitive therapy of substance abuse. New York, NY: Guilford Press; 1993.

3. Kozlowski LT, Pillitteri JL, Sweeney CT, Whitfield KE, Graham JW. Asking questions about urges or cravings for cigarettes. Psychol Addict Behav. 1996;10(4):248-60.

4. Marques AC, Seibel SD. O craving. In: Seibel SD, Toscano Jr., editors. Dependência de drogas. São Paulo: Atheneu; 2001, p. 239-48.

5. Sayette MA, Shiffman S, Tiffany S, Niaura R, Martin CS, Shadel W. The measurement of drug craving: methodological approaches to craving research. Addiction. 2000;95(Suppl 2):S189-210.

6. Araujo RB, Oliveira MS, Mansur MA. A validação brasileira do Questionnaire of Smoking Urges. Cad Saude Publica. 2006;22(10):109-18.

7. Araujo RB, Oliveira MS, Moraes JFD, Pedroso RS, Port, F, Castro MGT. Validação da versão brasileira do Questionnaire of Smoking Urges - Brief. Rev Psiq Clín. 2007;34(4):166-75.
8. Cox LS, Tiffany ST, Christen AG. Evaluation of the brief questionnaire of smoking urges (QSU-brief) in laboratory and clinical settings. Nicotine Tob Res. 2001;3:7-16.

9. Tiffany ST, Drobes DJ. The development and initial validation of a questionnaire on smoking urges. Br J Addict. 1991;86:1467-76.

10. Tiffany ST, Singleton E, Haertzen CA, Henningfield JE. The development of a cocaine craving questionnaire. Drug Alcohol Depend. 1993;34:19-28.

11. Franken IHA, Hendriks VM, Van den Brink W. Initial validation of two opiate craving questionnaires The Obsessive Compulsive Drug Use Scale and Desires of Drug Questionnaire. Addict Behav. 2002;27:675-85.

12. Singleton EG, Anderson LM, Heishman SJ. Reliability and validity of the Tobacco Craving Questionnaire and validation of craving-induction procedure using multiple measures of craving and mood. Addiction. 2003;98:1537-46.

13. Sussner B, Smelson DA, Rodrigues S, Kline A, Losonczy M, Ziedonis D. The validity and reliability of a brief measure of cocaine craving. Drug Alcohol Depend. 2006;83(3):233-7.

14. Silveira DX, Fernandes M, Silveira ED, Jorge MR. Cocaine craving questionnaire: assessing craving among cocaine users in Brazil. Psychiatry Res. 2006;142:257-9.

15. Ribeiro M, Dunn J, Sesso R, Dias AC, Laranjeira R. Causes of death among crack cocaine users. Rev Bras Psiquiatr. 2006;28(3):196-202.

16. Laranjeira R, et. al. Seguimento de usuários de crack após dois anos. Rev Bras Psiquiatr. 1998;47(5):233-6.

17. Mattos P, Segenreich D, Saboya E, Louzã M, Dias G, Romano M Adaptação transcultural da escala ASRS-18 (versão1.1) para avaliação do transtorno de déficit de atenção e hiperatividade em adultos para o português. Rev Psiquiatr Clín. 2006;33(4):188-94.

18. Pedroso RS, Oliveira MS, Araujo RB, Moraes JFD. Tradução, equivalência semântica e adaptação cultural do Marijuana Expectancy Questionnaire (MEQ). PsicoUSF. 2004;9(2):129-36.

19. Pedroso RS, Oliveira MS, Moraes JFD. Tradução, adaptação e validação da versão brasileira da escala Marijuana Expectancy Questionnaire. Cad Saude Publica. 2007;23(1):63-73.

20. Organização Mundial de Saúde. Classificação de transtornos mentais e de comportamento da CID-10: descrições clínicas e diretrizes diagnósticas. Porto Alegre: Artes Médicas; 1993.

21. Araujo RB, Oliveira M, Piccoloto LB, Szupszynski KPDR. Sonhos e craving em alcoolistas na fase de desintoxicação. Rev Psiq Clín. 2004;31(2):63-9.

22. Pasquali L. Princípios de elaboração de escalas psicológicas. Rev Psiq Clín. 1998;25(5):206-13.

23. Guimarães CF, Santos DVV, Freitas RC, Araujo RBl. Perfil do usuário de crack e fatores relacionados à criminalidade em unidade de internação para desintoxicação no Hospital Psiquiátrico São Pedro de Porto Alegre (RS). Rev Psiquiatr Rio Gd Sul. 2008;30(2):101-8.

24. Oliveira LG, Nappo SA. Crack na cidade de São Paulo: acessibilidade, estratégias de mercado e formas de uso. Rev Psiq Clín. 2008;35(6):212-8. 